\title{
Pequeñas y medianas empresas tecnológicas en México: distribución regional e inserción en cadenas globales de valor
}

\section{Mexican Small and Medium Technological Enterprises: Regional Distribution and their Insertion into Global Value Chains}

\author{
Óscar F. Contreras Montellano* (i) https://orcid.org/0000-0001-6000-5020 \\ Maciel García Fuentes ${ }^{\star *}$ (D) https://orcid.org/0000-0001-9337-7904
}

\section{Resumen}

El objetivo de este artículo es presentar una caracterización de las pequeñas y medianas empresas tecnológicas mexicanas y analizar los mecanismos por los cuales se insertan en segmentos de alto valor agregado en cadenas globales de valor. La metodología utilizada es de tipo mixto; incluye la creación de un directorio nacional de pequeñas y medianas empresas tecnológicas mexicanas, la aplicación de una encuesta por muestreo y la realización de entrevistas en profundidad. Entre los resultados relevantes destacan la distribución geográfica y sectorial de estas empresas (82\% están concentradas en I5 zonas metropolitanas con alta presencia de empresas multinacionales) y la identificación de los mecanismos de adquisición de las capacidades tecnológicas y empresariales que les permiten competir en segmentos de alto valor agregado. Una limitación de este trabajo deriva del análisis aún incipiente de la amplia base de datos generada por la investigación en la que está basado. Las conclusiones más importantes son dos: las pequeñas empresas tecnológicas mexicanas se concentran en las zonas con mayor flujo de inversión extranjera directa en el país, y los mecanismos de creación y escalamiento se relacionan con las derramas de conocimiento de las empresas multinacionales y con la maduración de algunos sistemas de innovación regional.

Palabras clave: PYME tecnológicas mexicanas; derramas de conocimiento; cadenas globales de valor; sistemas de innovación.

\section{Abstract}

This paper aims to present a characterization of Mexican small and medium technological enterprises, and to analyze the processes by which they inserted into higher value added segments in global value chains. The mixed methodology used includes the creation of a national directory, a sample survey, and conducting in-depth interviews. Results show the sectorial and geographic distribution of small and medium technological enterprises ( $82 \%$ are concentrated in 15 metropolitan areas with strong presence of multinational companies), and the mechanisms of acquisition of technological and managerial capacities which lead companies to compete in high value added segments. A limitation of this work derived from the still incipient analysis of the large database generated by the research on which it is based. The conclusions highlight two relevant findings: Mexican small and medium technological enterprises concentrate in regions with massive flows of foreign direct investment; and the mechanisms for the creation and upgrading of this type of companies are linked to knowledge spillovers from multinational companies and to the maturation of some regional innovation systems.

Keywords: small and medium Mexican technological enterprises; knowledge spillovers; global value chains; innovation systems.

Cómo citar: Contreras Montellano, O. F., y García Fuentes, M. (2019). Pequeñas empresas tecnológicas en México: distribución regional e inserción en cadenas globales de valor. región y sociedad, 3I, e I234. doi: I0.22 I 98/rys20 I 9/3 |/ 234

${ }^{*}$ Autor para correspondencia. El Colegio de la Frontera Norte, Departamento de Estudios Sociales. Km. 18.5, carretera escénica Tijuana-Ensenada, C. P. 22560, Tijuana, Baja California, México. Correo electrónico: ocontre@colef.mx

** Centro de Enseñanza Técnica y Superior (Cetys Universidad), Campus Tijuana, Escuela de Administración y Negocios. Calzada Cetys 813, Lago Sur, C. P. 22210, Tijuana, Baja California, México. Correo electrónico: maciel.garcia@cetys.mx 


\section{Introducción}

Con el Tratado de Libre Comercio de América del Norte (TLCAN), México se convirtió en uno de los países con mayor atracción de inversión extranjera directa (IED) en América Latina. Una parte importante de estas inversiones se canalizaron hacia el sector manufacturero, principalmente para la instalación de plantas de ensamble de empresas multinacionales (EMN) en industrias globalizadas, como la electrónica, la automotriz y la aeroespacial, entre otras, y para diversos segmentos del comercio y de los servicios. Así, a lo largo de los últimos 25 años, las EMN afianzaron su presencia y liderazgo sobre los segmentos más dinámicos del sector manufacturero y sobre algunos de los servicios de mayor alcance global. Hacia el año 2010 había más de 1700 EMN operando en México, las cuales empleaban alrededor de cinco millones de personas (Contreras y Carrillo, 2012).

Desde el inicio, la presencia masiva de EMN en México resultó un tema controversial. Frente al argumento de que las EMN generaban diversas derramas tecnológicas y de conocimiento sobre la economía nacional, tales como la trasferencia tecnológica y de capacidades gerenciales hacia las empresas locales, se objetó con frecuencia que tales derramas eran insignificantes y que el principal efecto de las EMN fue una alta polarización de la economía nacional, con segmentos modernos y globalizados (aquellos controlados por las EMN) y otros atrasados y de baja productividad (aquellos ligados al mercado interno).

Una de las críticas más persistentes y mejor documentadas al modelo de inserción global liderado por las EMN se refiere a la muy escasa participación de las pequeñas y medianas empresas locales (PYME) en las actividades económicas globalizadas (Bair y Gereffi, 2001; Frederick y Gereffi, 2011). Sin embargo, algunas investigaciones recientes han identificado diversos mecanismos a través de los cuales las EMN trasfieren conocimiento y facilitan la incorporación de las PYME a las cadenas globales de valor (CGV) (Contreras, Carrillo y García, 2017; Gereffi y Fernandez-Stark, 2016). Si bien se trata de un fenómeno reciente y de alcance limitado, su caracterización y análisis resulta de gran relevancia puesto que involucra, al parecer, el fenómeno más exitoso de creación de empresas locales mexicanas de alta tecnología incorporadas a las CGV.

Este trabajo tiene como objetivo caracterizar estas PYME tecnológicas y analizar sus mecanismos de inserción y escalamiento (upgrading) en segmentos de alto valor agregado en CGV. La hipótesis es que los principales mecanismos de formación y escalamiento de las PYME tecnológicas en México se relacionan con las derramas de conocimiento por parte de las EMN y con la maduración de algunos sistemas regionales de innovación (SRI). La definición operativa de PYME tecnológicas incluye dos subtipos de empresas: nuevas empresas de base tecnológica (cuyo principal activo es el capital intelectual) y empresas de servicios intensivas en conocimiento (que involucran actividades económicas cuya intención final es la creación, acumulación o diseminación de conocimiento).

El artículo está dividido en seis partes. Después de esta introducción, en el segundo apartado, se presenta un breve recuento de la convergencia entre dos enfoques teóricos que resultan pertinentes para el análisis de las PYME tecno- 
lógicas en México; en el tercer apartado se describe la metodología utilizada en el estudio y el alcance de los resultados presentados en el artículo; en el cuarto y quinto apartados se presentan los principales hallazgos: la estimación del número de PYME tecnológicas en México, su distribución sectorial y regional, y dos patrones diferenciados de formación y escalamiento en las CGV; por último, en la sección de conclusiones, se resumen los hallazgos y se formulan dos escenarios derivados de la eventual ratificación o rechazo del Tratado entre México, Estados Unidos y Canadá (T-MEC) -el acuerdo resultante de la renegociación del TLCAN-.

\section{Derramas de conocimiento y aprendizaje tecnológico de las PYME}

El análisis de la creación y desempeño de las PYME de base tecnológica ha cobrado relevancia a lo largo de las últimas dos décadas, sobre todo en el marco del análisis de los emprendimientos de alta tecnología en países desarrollados (Klepper y Thompson, 2005; Muller y Doloreux, 2009). En el caso de países emergentes, en especial de América Latina, se trata de un tema prácticamente inexplorado; si bien algunos trabajos han abordado determinados aspectos de este fenómeno, se trata de excepciones en el marco de una narrativa centrada en las limitaciones de las PYME para incursionar en segmentos de alta tecnología (Bianchi, Glavas y Mathews, 2017; Vera-Cruz y Dutrénit, 2005; Zevallos, 2003). Debido a las limitaciones de los entornos en los que operan las PYME de países emergentes, así como sus dificultades para acceder a los mercados internacionales o a las cadenas de suministro de las empresas multinacionales (EMN), el estudio de su formación y desempeño se ha beneficiado de dos perspectivas analíticas convergentes: las cadenas globales de valor (CGV) y los sistemas de innovación (SI).

Durante las últimas décadas el enfoque CGV ha adquirido una amplia influencia en los estudios sobre los vínculos entre empresas que operan en mercados internacionales, en particular cuando dichas relaciones involucran diversos eslabones de un mismo proceso de producción y distribución de alcance global. En sus inicios, las investigaciones realizadas bajo este enfoque centraron su atención en el papel de las grandes empresas que comandaban tanto la coordinación como la integración funcional de múltiples establecimientos productivos y de servicios dispersos en diferentes regiones y países (Gereffi y Kaplinsky, 2001; Gereffi y Korzeniewicz, 1994; Pietrobelli y Rabellotti, 2006). En América Latina la aplicación de la perspectiva CGV se ha orientado principalmente al análisis de los procesos de escalamiento (upgrading), en busca de los determinantes y mecanismos de mejora en empresas, regiones y sectores, tratando de explicar cómo las empresas nacionales y locales pueden participar en los mercados globales y mejorar su productividad, al tiempo que desarrollan capacidades para producir bienes y servicios de mejor calidad y mayor valor agregado. Los tipos de escalamiento identificados son cuatro: 1) de producto; 2) de proceso; 
3) funcional, cuando se incorporan nuevas funciones de mayor valor agregado; e 4) intersectorial, cuando se aplican las competencias adquiridas en determinada industria o sector para transitar a un nuevo sector intensivo en capital y tecnología (Giuliani, Piertobelli y Rabellotti, 2005; Humphrey y Schmitz, 2004). Uno de los resultados más consistentes en diversas investigaciones es que en América Latina los procesos de escalamiento son por lo general de producto y de proceso, como resultado del aprendizaje tecnológico, y son poco frecuentes los casos de escalamiento funcional e intersectorial (Dini y Stumpo, 2011; Piertobelli y Rabellotti, 2006).

En el análisis del escalamiento resulta clave el concepto de gobernanza de la CGV. Éste se refiere a la trama de las "relaciones de poder y autoridad que determinan cómo se asignan los recursos humanos, materiales y financieros, y su influencia en la cooperación de las empresas a lo largo de la cadena" (Gereffi, 1994, p. 97). En la configuración de la gobernanza en la CGV intervienen las capacidades técnicas de los agentes, pero también su posición de poder en el interior de la cadena. Son tres los parámetros que definen tal configuración: 1) la complejidad involucrada en la trasferencia de conocimiento e información, dadas las especificaciones de producto y proceso; 2) la factibilidad de codificación de la información y conocimientos trasferidos, y 3) la capacidad de los proveedores para cumplir con los requisitos explícitos e implícitos demandados por las transacciones (Gereffi, Humphrey y Sturgeon, 2005).

Para el caso de las PYME en América Latina, y en general en economías emergentes, el enfoque CGV resulta de gran utilidad, especialmente en el marco de la creciente tensión entre dos orientaciones divergentes: la tendencia a la segmentación internacional de los procesos productivos y los servicios, por una parte, y las renovadas políticas de protección de las economías nacionales, por la otra. En la lógica de la segmentación, que ha sido la tendencia predominante durante las últimas décadas, las EMN trasfieren conocimientos técnicos y prácticas gerenciales a algunos proveedores de los países donde operan, de tal modo que estas empresas locales sean capaces de cumplir con los estándares de calidad y tiempos de entrega requeridos, lo cual reduce costos de producción y problemas logísticos (Contreras, 2016; Yuhua, 2014). Ejerciendo una posición dominante en la gobernanza de la cadena, las EMN necesitan proveedores confiables en los cuales delegar no sólo los componentes, procesos o servicios básicos, sino también algunos de creciente complejidad y valor agregado.

Por otra parte, el dominio de las capacidades para convertirse en proveedores de las EMN no depende sólo de los propios recursos de las PYME, o de la trasferencia directa de capacidades por parte de las EMN, ya que para la adecuada asimilación de conocimientos, tecnología y habilidades por parte de las empresas locales se requieren ciertas condiciones en el entorno económico, social e institucional en el que operan. Aspectos como la calidad de la infraestructura y de las comunicaciones, la educación de los técnicos e ingenieros, la información y los servicios gubernamentales pueden facilitar o inhibir las capacidades de absorción de las PYME (Dini y Stumpo, 2004). En la intersección entre las exigencias del mercado global, con sus presiones competitivas y requerimientos técnicos, y las características del entorno local, que condicionan el desempeño 
de las PYME y su eventual escalamiento hacia segmentos de mayor valor agregado, la perspectiva de los SI cobra una particular relevancia.

Originalmente el enfoque SI se desarrolló como un marco conceptual anidado en la escala nacional y dio lugar a la noción de sistema nacional de innovación (SNI) (Freeman, 1987; Lundvall, 1992). Después se conceptualizaron otras escalas para caracterizar sistemas regionales de innovación (SRI), sistemas sectoriales de innovación (SSI) y sistemas transfronterizos de innovación (STI) (Cooke, 1992; Malerba y Nelson, 2011; Trippl, 2006). ${ }^{1}$

Independientemente de la escala territorial que se privilegie en el análisis, el enfoque SI se basa en la premisa de que tanto el aprendizaje tecnológico como la innovación se desarrollan en el marco de una compleja trama de aprendizaje interactivo entre diversos agentes, y no están determinados por completo por relaciones de mercado impulsadas por el precio. La innovación, desde este punto de vista, no se genera en empresas aisladas o en relaciones bilaterales cliente/proveedor, sino en una red de interacciones continuas con múltiples agentes en el territorio. Además, los procesos de innovación no sólo incluyen las nuevas tecnologías, sino que también comprenden las innovaciones de productos y procesos, así como otras formas de innovación no tecnológicas, como las desarrolladas en las organizaciones de servicios (Lundvall, 2007). Ya sea que se refiera a la escala nacional, regional, local o trasfronteriza, la perspectiva SI enfatiza la importancia de las trayectorias tecnológicas y de los activos institucionales en los procesos de aprendizaje colectivo, lo que otorga una alta capacidad explicativa al entorno institucional que estimula o inhibe el aprendizaje tecnológico y la innovación.

Uno de los supuestos centrales de este enfoque plantea que el conocimiento involucrado en los procesos de innovación, y en general en el desarrollo económico de regiones y países, está territorialmente localizado; se trata de un activo intangible que no puede reubicarse con la misma facilidad que la maquinaria, puesto que algunos de los componentes más importantes del conocimiento están incorporados en las mentes y cuerpos de los agentes, en forma de rutinas organizacionales y relaciones entre personas y organizaciones. En otras palabras, para la perspectiva SI los procesos de aprendizaje tecnológico y de innovación están territorialmente localizados, socialmente incrustados, y sólo pueden ser analizados como producto de la interacción entre agentes diversos (Lundvall, 2007).

En el enfoque CGV, la gobernanza, el escalamiento y la trasferencia de conocimiento a lo largo de la cadena son conceptos clave (Gereffi y Fernandez-Stark, 2011); por su parte, el enfoque SI privilegia los conceptos de aprendizaje interactivo e innovación conjunta entre empresas e instituciones (Lundvall, 2007). Ambas perspectivas otorgan una gran importancia a los procesos de mejora, si bien el enfoque SI otorga una importancia fundamental a la construcción de capacidades de absorción en el nivel meso y macro, mientras que el análisis CGV se centra en procesos de escalamiento en el nivel micro y meso (Gereffi, Humphrey y Sturgeon, 2005).

1 En este artículo, el enfoque de los sistemas regionales de innovación (SRI) se considera como una variante de la teoría de los sistemas de innovación (SI). 
La convergencia de ambos enfoques puede resultar de gran utilidad para la investigación empírica de las PYME tecnológicas en México, ya que la afluencia de EMN a diversas regiones del país ha propiciado la interacción de estas empresas globales con muy diversos agentes locales y regionales, con la consecuente diversidad de acoplamientos de los actores económicos e institucionales a los desafíos y oportunidades generados por la presencia de las EMN. La integración de estos dos enfoques en una plataforma conceptual común no es un esfuerzo inédito; se han realizado algunos avances al intentar caracterizar la interacción entre los sistemas de innovación (a escala nacional, regional o local) con diferentes patrones de gobernanza de la CGV que en conjunto influyen en los mecanismos de aprendizaje e innovación de las empresas. Se pueden identificar al menos cuatro dimensiones de convergencia entre los enfoques de CGV y de sistemas regionales de innovación (SRI): 1) procesos de aprendizaje interactivo entre empresas y co-evolución entre empresas e instituciones; 2) procesos de escalamiento a partir de aprendizaje tecnológico y políticas públicas; 3 ) procesos de creación de capacidades de absorción a partir de políticas y agencia, y 4) la interacción entre empresas y otros actores clave del sistema como factor crucial para la trasferencia de conocimiento, el escalamiento y la innovación (Cooke, Uranga y Etxebarria, 1997; Gereffi, Humphrey y Sturgeon, 2005).

En algunos trabajos se argumenta que la perspectiva SI puede fortalecerse si se trasciende el alcance nacional o subnacional en el que por lo general se ha confinado y se incorpora en el análisis la dimensión de intercambio y colaboración internacional para la generación y difusión de conocimiento e innovación; así mismo el enfoque CGV podría enriquecerse al incluir el contexto organizacional e institucional, así como la influencia de las políticas de innovación sobre las formas de gobernanza en la CGV y sobre los procesos de aprendizaje y escalamiento (Humphrey y Schmitz, 2004; Jurowetzki, Lema y Lundvall, 2018; Pietrobelli y Rabellotti, 2006).

En el mismo sentido, Pietrobelli y Rabellotti (2011) argumentan que es posible articular ambos enfoques para discernir la forma en que los sistemas de innovación (a escala nacional, regional o local) y los diferentes patrones de gobernanza de la cadena global de valor, influyen de forma conjunta en los mecanismos de aprendizaje e innovación de las empresas. Por su parte, Jurowetzki, Lema y Lundvall (2018) identifican diversas convergencias y traslapes, pero poco diálogo, entre las perspectivas CGV y SI, y plantean que su integración sería de utilidad para mejorar el conocimiento sobre procesos socioeconómicos en países en desarrollo, así como en la construcción de una base de conocimiento útil para la acción.

A pesar de algunas diferencias teóricas y metodológicas entre estas dos perspectivas, al parecer pueden complementarse en el marco de la tradición de los estudios que abordan los fenómenos económicos a partir de las herramientas conceptuales de la sociología (Smelser y Swedberg, 2005). En términos teóricos, las perspectivas CGV y SI comparten el estatus de teorías de alcance medio, es decir, teorizaciones con un nivel intermedio de abstracción, a medio camino entre las teorías generales y las proposiciones empíricas. En términos metodológicos, su valor reside en que plantean conjuntos limitados de supues- 
tos que pueden ser confirmados por la investigación empírica mediante hipótesis específicas, pero a la vez son suficientemente abstractas para abordar diferentes esferas de la acción y de la estructura social, por lo que trascienden la simple descripción o la generalización; en el plano de la propia teoría, los supuestos e hipótesis se conectan con redes más amplias de teorías y permiten la ampliación y sistematización de la teoría en un marco de alta sensibilidad a la evidencia empírica (Merton, 1968; Portes, 2004). Lundvall (2007) argumenta que la perspectiva SI constituye un "dispositivo de enfoque" equivalente a una teoría, que permite observar, entender y controlar fenómenos que de otro modo no podrían ser captados; ayuda a organizar y enfocar el análisis, a explicar determinados segmentos de la realidad y a proveer bases para la acción.

\section{Metodología}

Los estudios sobre las PYME tecnológicas en México son recientes, en buena medida porque el fenómeno mismo es relativamente nuevo: la mayoría de estas empresas fueron creadas durante los últimos 25 años, es decir, en el periodo del TLCAN. En particular, los estudios que han abordado la relación entre las EMN y las empresas locales han identificado cuatro modalidades de trasferencia de conocimiento: 1) el desarrollo de vínculos verticales, entre ellos las relaciones entre cliente y proveedor; 2) la emergencia de procesos de demostración/ imitación como resultado de la operación de EMN en contextos regionales y locales; 3 ) el entrenamiento y capacitación de las EMN a sus empleados, quienes al cambiar de empleo pueden llevar los conocimientos adquiridos a otras empresas o utilizarlos como base para crear sus propios negocios, y 4) el establecimiento de centros de investigación, desarrollo e innovación de filiales de EMN en México (Carrillo y Lara, 2005; Carrillo y Zarate, 2004; Casalet y González, 2004; Contreras, 2000; Contreras y Hualde, 2012; Contreras e Isiordia, 2010; Dussel, 2012; Fuentes y Dutrénit, 2008; Hualde y Gomis, 2007; Vera-Cruz y Dutrénit, 2005; Villavicencio y Arvanitis, 2001).

Entre los principales mecanismos de formación de PYME tecnológicas identificados en las investigaciones empíricas, están los procesos de spin-off empresariales, es decir, casos en los que algunos exempleados de EMN formaron una PYME y después lograron integrarse con éxito en cadenas de proveeduría de las EMN (Contreras, 2000; Contreras e Isiordia, 2010; Fuentes y Dutrénit, 2008; Vera-Cruz y Dutrénit, 2005), y los nuevos emprendimientos tecnológicos ligados a las instituciones y organizaciones de los SRI, que con frecuencia utilizan recursos provenientes del Consejo Nacional de Ciencia y Tecnología (CONACYT), así como de diversos programas estatales y federales (Casalet, Buenrostro y Becerril, 2009; López de Alba, 2014; Valenzuela y Bracamonte, 2014).

Una de las limitaciones más comunes de esas investigaciones consiste en la selección de un reducido número de casos para hacer inferencias analíticas. Si bien la cuidadosa selección de los casos, basada en estrategias conceptualmente robustas, ha permitido identificar y caracterizar algunos de los mecanismos involucrados en la formación y escalamiento de las PYME tecnológicas en ac- 
tividades o regiones específicas, con dificultad se pueden identificar patrones generales o elaborar tipologías exhaustivas a partir de esas metodologías. Hasta ahora, la discusión académica sobre el tema ha estado basada principalmente en estudios de caso en actividades como la industria automotriz, los maquinados industriales y el software (Contreras, 2016; Contreras, Carrillo y Alonso, 2012), o en estudios de corte cuantitativo pero de alcance local (Fuentes y Dutrénit, 2008; Mendoza y Valenzuela, 2014).

Para la investigación en la que se basa el presente trabajo, se diseñó una estrategia más amplia y sistemática, que partió de la elaboración de un directorio de empresas tecnológicas localizadas en las 60 zonas metropolitanas (ZM) de México. ${ }^{2}$ Utilizando el Sistema de Clasificación Industrial de América del Norte (SCIAN), publicado por el Instituto Nacional de Estadística y Geografía (INEGI, 2014), ${ }^{3}$ se identificaron las clases industriales (a 6 dígitos) que en la literatura académica se consideran como intensivas en conocimiento o de base tecnológica. El procedimiento para identificar las clases pertinentes del SCIAN comprendió tres pasos: 1) análisis crítico de la literatura en torno a la clasificación de actividades intensivas en conocimiento y de base tecnológica (Alarcón y Díaz, 2016; Heckler, 2005; Kile y Phillips, 2009); 2) validación y ajuste de la primera selección en reuniones con investigadores, empresarios y consultores expertos familiarizados con este tipo de empresas en México y América Latina; y 3) búsquedas piloto en la base de datos del Directorio Estadístico Nacional de Unidades Económicas (DENUE) en su última actualización de 2018 (INEGI, 2018), focalizadas en las clases preseleccionadas. Como resultado de este procedimiento, se obtuvieron 45 clases pertenecientes a siete sectores; ${ }^{4}$ esto arrojó un listado de 2058 empresas correspondientes a esas clases, mismo que constituye el universo de la investigación. El procedimiento de selección basado en esas 45 clases de actividad garantiza que no se incluyeran empresas dedicadas a actividades de bajo contenido tecnológico.

Con base en ese directorio de alcance nacional, se calculó una muestra aleatoria para aplicar un instrumento estandarizado, con el objetivo de generar información sobre: el perfil de las empresas y los empresarios; los mecanismos de entrada al mercado; los procesos de aprendizaje; la formación de capacidades; los vínculos de las PYME con las EMN y con los SRI, y el escalamiento en la cadena de valor. El cuestionario contiene 73 preguntas y fue aplicado entre septiembre y noviembre de 2018 sobre una muestra de propietarios de empresas pertenecientes a alguna de las 45 clases industriales seleccionadas del SCIAN y localizadas en cuatro de las ZM correspondientes al universo de estudio: dos

2 Oficialmente se reconocen 59 zonas metropolitanas en México (Secretaría de Desarrollo Social, [SEDESOL], Consejo Nacional de Población [CONAPO] e Instituto Nacional de Estadística y Geografía [INEGI], 2012), pero incluimos Hermosillo, que en la actualidad se encuentra en proceso de reconocimiento.

3 EI SCIAN contiene 20 sectores, 94 subsectores, 305 ramas, 614 subramas y 1059 clases.

4 Sectores del SCIAN seleccionados y número de clases incluidas por sector: 11, agricultura, cría y explotación de animales, aprovechamiento forestal, pesca y caza (cuatro clases); 21, minería (dos clases); 31-33, industrias manufactureras (27 clases); 51, información en medios masivos (dos clases); 54, servicios profesionales, científicos y técnicos (ocho); 56 , servicios de apoyo a los negocios y manejo de residuos y desechos, y servicios de remediación (uno); 81, otros servicios, excepto actividades gubernamentales (uno). En cuanto al tamaño de las empresas, medido por personal ocupado, se seleccionaron los rangos: 6-10, 11-30, 31-50 y 51-100 empleados. 
ZM localizadas sobre la franja fronteriza entre México y Estados Unidos (Tijuana y Ciudad Juárez) y dos ZM localizadas en el interior de estados fronterizos (Hermosillo y Monterrey). ${ }^{5}$ Además, se realizaron 37 entrevistas en profundidad (incluyendo visitas a las plantas) para ahondar en aspectos cualitativos de las trayectorias de los empresarios y las empresas. En este artículo se reportan los resultados del análisis del directorio nacional de empresas PYME tecnológicas y del análisis de los estudios de caso.

\section{Resultados}

\section{El TLCAN y las PYME tecnológicas en México}

El flujo masivo de IED durante el periodo del TLCAN, así como el establecimiento de cientos de EMN en el país, procedentes sobre todo de Estados Unidos, pero también de Europa y Asia, dieron un impulso decisivo a la participación de México en la manufactura de bienes de tecnología intermedia y avanzada para los mercados globales. A pesar de ello, diversos obstáculos inhibieron la incorporación de las PYME locales como proveedoras en las CGV, entre ellos: 1) las limitaciones de las PYME para cumplir con los estándares de calidad, volumen y plazos exigidos por las EMN; 2) las políticas de compra de las EMN, las cuales privilegiaban a sus socios y proveedores globales más que a las empresas locales y 3) la ausencia de una política industrial que promoviera la creación de capacidades tecnológicas y empresariales en empresas locales (Contreras, Carrillo y Alonso, 2012; Vera-Cruz y Dutrénit, 2005).

Numerosas investigaciones han constatado la muy escasa participación de proveedores locales de alto nivel en las cadenas de suministro de las industrias globalizadas. Algunos trabajos recientes, sin embargo, revelan la presencia de una nueva generación de PYME intensivas en conocimiento incorporadas a las redes de proveedores de las EMN, la mayoría de ellas creadas en el periodo del TLCAN. Dichas PYME proveen diversos tipos de productos y servicios de alto valor agregado (Contreras, 2016; Contreras y García, 2017; Fuentes y Dutrénit, 2008). Si bien su número es reducido y su papel en las cadenas de valor es modesto, su presencia representa un fenómeno novedoso y de gran importancia para la comprensión de los mecanismos de formación y consolidación de este tipo de empresas. ${ }^{6}$

5 Las características técnicas de la metodología utilizada son las siguientes: población objetivo: PYME mexicanas pertenecientes a 45 clases industriales en los sectores SCIAN 11, 21, 31-33, 51, 54, 56 y 81. Tamaño de la muestra: 127 empresas distribuidas representativamente en las 4 zonas metropolitanas ( 35 en Tijuana, 30 en Ciudad Juárez, 30 en Hermosillo y 32 en Monterrey). Nivel de confianza: $95 \%(Z=1.96)$. Error muestral $\pm 7 \%$. Instrumentos aplicados: a) encuesta aplicada en sitio o en línea; b) estudio de caso múltiple con empresas seleccionadas a partir del directorio.

6 Desde luego, no todas las PYME tecnológicas mexicanas están asociadas a las CGV ni a desprendimientos tipo spin off. Sin embargo, de acuerdo con Contreras (2016) y Contreras y García (2017), el vínculo con las CGV y los desprendimientos tipo spin off han sido los principales determinantes para la formación de PYME tecnológicas mexicanas durante los últimos 25 años. Como se muestra en la sección de resultados, el presente estudio abarca a todas las PYME tecnológicas mexicanas, incluyendo a aquellas que evolucionaron desde actividades tradicionales. 
Utilizando como fuente el DENUE (INEGI, 2018), y con base en la metodología reseñada en la sección anterior, identificamos 2058 PYME tecnológicas en México, es decir, pequeñas empresas pertenecientes a alguna de las 45 clases del SCIAN clasificadas como intensivas en conocimiento o de base tecnológica, a lo largo de las 60 zonas metropolitanas del país. En la figura 1 se observa que las PYME tecnológicas se concentran en dos franjas, una en la región norte del país (en particular en las ZM de Tijuana, Hermosillo, Ciudad Juárez, Chihuahua, La Laguna, Saltillo y Monterrey) y otra en el centro-occidente de México (sobre todo en las ZM del Valle de México, Querétaro, Puebla, León, Aguascalientes, San Luis Potosí y Guadalajara). ${ }^{7}$ En estas 15 zonas metropolitanas se concentra 82\% de las PYME tecnológicas que operan en México. Más aún, las ZM del Valle de México, Monterrey y Guadalajara concentran $49.3 \%$ del total de empresas tecnológicas del país.

Con respecto a la franja norte, conviene mencionar que la IED anual en los estados involucrados ha tenido un incremento sostenido durante las últimas décadas; ha pasado de 750 millones de dólares (MDD) en 1989, a 12500 MDD en 2018 (INEGI, 2019), un monto 15 veces mayor, debido sobre todo a la oleada masiva de inversiones desatada por el TLCAN. Del total nacional, la franja norte concentró 39\% de la IED en 2018; fueron Baja California, Sonora, Chihuahua, Coahuila, Nuevo León y Tamaulipas los estados más dinámicos (Secretaría de Economía [SE], 2019). En estas entidades, un factor determinante en la formación y escalamiento de las PYME tecnológicas mexicanas ha sido la localización y evolución de la industria manufacturera, maquiladora y de servicios de exportación (IMMEX), sobre todo en las últimas dos décadas, con la llegada de EMN pertenecientes a sectores de alta tecnología (Contreras, Carrillo y García, 2017).

En cuanto a la franja de la región centro-occidente, los estados involucrados concentraron aproximadamente 50\% de la IED en 2018 (de la cual 20\% tuvo lugar en los estados de occidente y $30 \%$ en los del centro) (SE, 2019). Al igual que en la franja norte, en la centro-occidente los flujos de IED se multiplicaron a raíz del TLCAN, en este caso canalizados principalmente hacia las industrias de autopartes-automotriz, aeroespacial y electrónica, que se han consolidado como segmentos de gran dinamismo en el proceso de especialización productiva de estas entidades. A lo largo de las últimas décadas, los constantes flujos de IED en actividades intensivas en conocimiento, lideradas sobre todo por EMN, han dado lugar a diversas derramas tecnológicas y de conocimiento que, así sea de manera limitada y sin el acompañamiento de programas de incentivos, han contribuido a la formación de spin-offs empresariales creados por emprendedores mexicanos.

Respecto a su distribución sectorial, 49\% de estas PYME pertenecen al sector de servicios profesionales, científicos y técnicos (sector 54 del SCIAN) y $33.4 \%$, a la industria manufacturera (sectores 31-33). Destacan por su alta participación

7 La identificación y análisis de las PYME tecnológicas se basa en las zonas metropolitanas referidas en la sección anterior. Su agrupación en dos "franjas" regionales tiene como propósito mostrar el contraste en la especialización sectorial de la IED y su correspondencia con la actividad de las PYME tecnológicas creadas durante el periodo del TLCAN. 


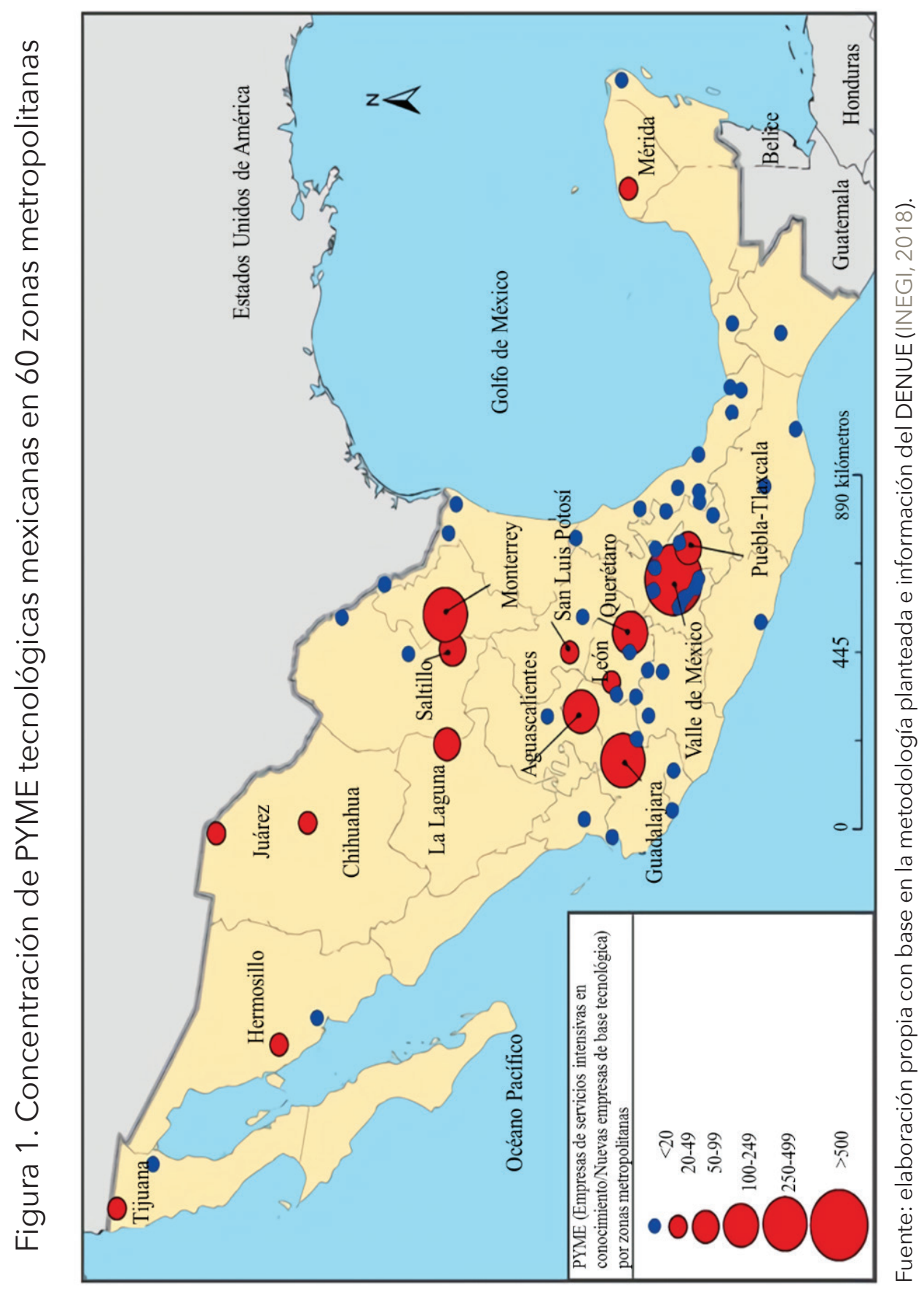


las clases 541510 (servicios de diseño de sistemas de cómputo y servicios relacionados), con 23.6\%, 332710 (maquinado de piezas metálicas para maquinaria y equipo en general), con 20.9\%, 541330 (servicios de ingeniería) y 541380 (laboratorio de pruebas), con $8.2 \%$ y $7.6 \%$, respectivamente.

Estas cifras de PYME tecnológicas, así como su distribución sectorial, guardan una clara correspondencia con las trayectorias productivas de las respectivas regiones y con los flujos de IED durante los años recientes. Así, por ejemplo, en las zonas metropolitanas del norte del país, como Tijuana, Hermosillo, Ciudad Juárez y Monterrey, predominan las clases 332710, 333999, 333249, 333510 y 331510 , que corresponden a servicios de automatización y manufactura avanzada (fabricación de piezas de precisión, diseño y fabricación de maquinaria, y equipos de prueba) para EMN de sectores consolidados, como el de electrónica, el automotriz y de dispositivos médicos, mientras que en la franja centro-occidente, en las zonas metropolitanas de Querétaro, Guadalajara, Puebla y Valle de México predominan las clases 541510, 511210, 511210, 332710, 336370 y 541330 (servicios de ingeniería y consultoría, desarrollo de software, fabricación de piezas troqueladas para vehículos automotrices y maquinados de piezas metálicas), las cuales se relacionan con la IED en los sectores automotriz y aeroespacial.

Dos patrones de creación y escalamiento de PYME mexicanas intensivas en conocimiento

En estudios anteriores (Contreras e Isiordia, 2010; Contreras, 2016) se encontró que las PYME que con mayor frecuencia se convirtieron en proveedoras de las EMN son empresas dedicadas a actividades de metalmecánica (MM), de tecnologías de la información (TI) y de logística (LG). La investigación en la que se basa este trabajo permitió detectar además un número significativo de empresas de servicios de ingeniería, automatización, manufactura avanzada y metrología incorporadas a la base de proveedores de las EMN.

Las PYME de cada una de estas actividades tienen un perfil específico, pero es notable el contraste entre las empresas creadas mediante desprendimientos tipo spin-off frente a aquellas que se originaron mediante emprendimientos o start-ups. Los propietarios de empresas spin-off tienen mayor escolaridad, mayor experiencia laboral previa a la creación de su negocio, y mayor vínculo previo con EMN. En relación con la escolaridad, es notable el hecho de que 63\% de los propietarios de spin-offs tienen estudios de posgrado, frente a $37 \%$ de los dueños de start-ups. Respecto de la experiencia laboral previa, los propietarios de empresas creadas mediante spin-off tienen un promedio de 2.7 empleos previos, frente a 2.2 de los empresarios de start-ups, pero además una experiencia laboral que prácticamente duplica el tiempo de trabajo en otras empresas, previa a la creación de su negocio. De manera consistente, 59\% de los emprendedores de spin-offs tuvieron como último empleo previo un puesto en una EMN, frente a sólo $11 \%$ de los dueños de start-ups. En cuanto a las empresas mismas, el vínculo previo con las EMN parece ser decisivo en la conformación de las oportunidades de mercado, ya que $82 \%$ de los spin-offs tienen como prin- 
cipal cliente actual a una EMN, mientras que solo $45 \%$ de los start-ups están en esa situación.

Con base en los estudios de caso en las ciudades de Tijuana, Ciudad Juárez, Hermosillo y Monterrey, se pueden identificar dos trayectorias estilizadas en la consolidación de las PYME intensivas en conocimiento que se han convertido en proveedoras de EMN.

Acumulación de capacidades tecnológicas

y de gestión mediante vínculos con EMN

Este tipo de trayectoria corresponde principalmente a empresas industriales y de servicios tecnológicos creadas mediante desprendimientos tipo spin-off por exempleados de EMN (técnicos, ingenieros o gerentes) después de diversas experiencias de trabajo en empresas industriales o de servicios tecnológicos. Una parte importante de esas experiencias trascurrieron en empresas multinacionales.

En algunos casos las empresas agrupadas en este tipo de trayectoria no fueron creadas por desprendimientos tipo spin-off, pero en una etapa temprana establecieron una relación de proveeduría con una EMN; en tales casos, con frecuencia el primer paso para acceder a la base de proveedores de las EMN consiste en la obtención de contratos de corto plazo mediante contactos con empresas que son clientes o proveedores de las EMN, y sólo en casos excepcionales el contacto se realiza a través del mercado abierto. Sea cual fuese el origen del contacto con la EMN, es muy común que los primeros contratos con empresas multinacionales se generen a partir de situaciones de emergencia en el proceso de producción (por ejemplo, descomposturas en los equipos o necesidad de subcontratar debido a pedidos inusuales) o bien para externalizar servicios periféricos de bajo costo.

En una segunda etapa, la empresa local logra consolidar su posición como proveedora a partir de la ejecución de un "proyecto crítico" para una EMN, es decir, una intervención planeada y no en una situación de emergencia, con el fin de ejecutar tareas de mantenimiento de equipos, desarrollo de software a la medida o externalización de servicios especializados. En la realización de un proyecto de ese tipo, la EMN puede evaluar las capacidades técnicas de la PYME, así como su nivel de respuesta bajo presión y su compromiso. De este modo, la PYME se convierte en proveedora confiable en proyectos de largo plazo y de mayor complejidad técnica. Las empresas creadas a través de spin-offs tienden a entrar en la cadena de suministros en esta etapa, ya que su reputación se basa en primera instancia en el desempeño previo de los propietarios como empleados de las multinacionales.

En una tercera etapa, las empresas PYME invierten en infraestructura y equipamiento para satisfacer una demanda más estable o de mayor complejidad por parte de las EMN. Se convierten en proveedoras permanentes de servicios tecnológicos, incluidos proyectos para el diseño y la fabricación de nuevos equipos industriales. Esta trayectoria de formación y escalamiento se representa en la figura 2. 
Figura 2. Desarrollo de capacidades tecnológicas en interacción con EMN

\begin{tabular}{|c|c|c|c|}
\hline Origen & Inicio & Consolidación & Escalamiento en la cadena de valor \\
\hline \multirow[t]{3}{*}{$\begin{array}{l}\text { Ingenieros con } \\
\text { experiencia } \\
\text { laboral en EMN } \\
\text { (10 años) }\end{array}$} & & & $\begin{array}{l}\text { Proveedores permanentes de } \\
\text { servicios tecnológicos, } \\
\text { incluyendo diseño y fabricación } \\
\text { de maquinaria y equipo } \\
\text { en coordinación técnica y } \\
\text { administrativa con las EMN }\end{array}$ \\
\hline & & $\begin{array}{l}\text { Proyecto crítico exitoso } \\
\text { las convierte en } \\
\text { proveedores } \\
\text { permanentes, bajo } \\
\text { supervisión de la EMN }\end{array}$ & \\
\hline & $\begin{array}{l}\text { Proveedor eventual } \\
\text { para solución de } \\
\text { contingencias de la } \\
\text { EMN }\end{array}$ & & \\
\hline
\end{tabular}

Fuente: elaboración propia.

Desarrollo de capacidades tecnológicas y asociativas en interacción con instituciones regionales

La segunda trayectoria estilizada corresponde principalmente a empresas de servicios tecnológicos (desarrollo de software, automatización, metrología, consultoría tecnológica, integración de sistemas y dispositivos móviles) creadas mediante emprendimientos, con pocos o ningún vínculo previo con EMN. Se trata de negocios creados por profesionales formados en diversas disciplinas de la ingeniería. Con frecuencia estos empresarios invierten sus ahorros personales o familiares, a veces asociados con amigos, para crear una PYME orientada al mercado local o regional. A diferencia del perfil anterior, por lo general sus primeros clientes son otras empresas locales o nacionales, además de ofertar diversos servicios para el mercado abierto, y sólo en una etapa posterior llegan a convertirse en proveedoras de una o más EMN. Un patrón recurrente en estos casos consiste en la realización exitosa de un proyecto de especial relevancia para una EMN en una coyuntura crítica, después de lo cual se convierten en proveedoras confiables y permanentes. 
Estas PYME suelen mantener, desde el inicio, vínculos con las asociaciones empresariales regionales y nacionales (sobre todo aquellas relacionadas con las $\mathrm{TI})$, así como con los diversos esquemas asociativos, como clústeres, lo que les permite un buen nivel de conocimiento acerca de los programas gubernamentales para las PYME. Algunos de estos empresarios conocen o han tenido apoyos del Programa para el Desarrollo de la Industria del Software (PROSOFT) de la Secretaría de Economía, el principal instrumento del gobierno mexicano para el impulso de esta industria. En una segunda etapa la empresa se consolida y es capaz de ofrecer productos o servicios de alto valor agregado a través de: a) la asociación con otras pequeñas empresas locales, y b) teniendo acceso a los fondos gubernamentales a fin de fortalecer sus capacidades técnicas, ya sea adquiriendo nuevos equipos o mediante el desarrollo de proyectos específicos. Algunas de las nuevas empresas creadas en diversas incubadoras de empresas regionales se insertan de manera directa en esta etapa, por lo general lanzando al mercado productos o servicios innovadores desde el inicio.

En la tercera etapa la empresa PYME accede a nichos especializados, con frecuencia en productos o procesos vinculados con CGV. En esta etapa las empresas locales tienden a asociarse con otras PYME (a veces mediante empresas integradoras), así como a gestionar fondos gubernamentales y a acceder a diversos programas de estímulo a la innovación. La trayectoria descrita se representa en la figura 3.

Figura 3. Desarrollo de capacidades técnicas, de asociación y uso de fondos gubernamentales

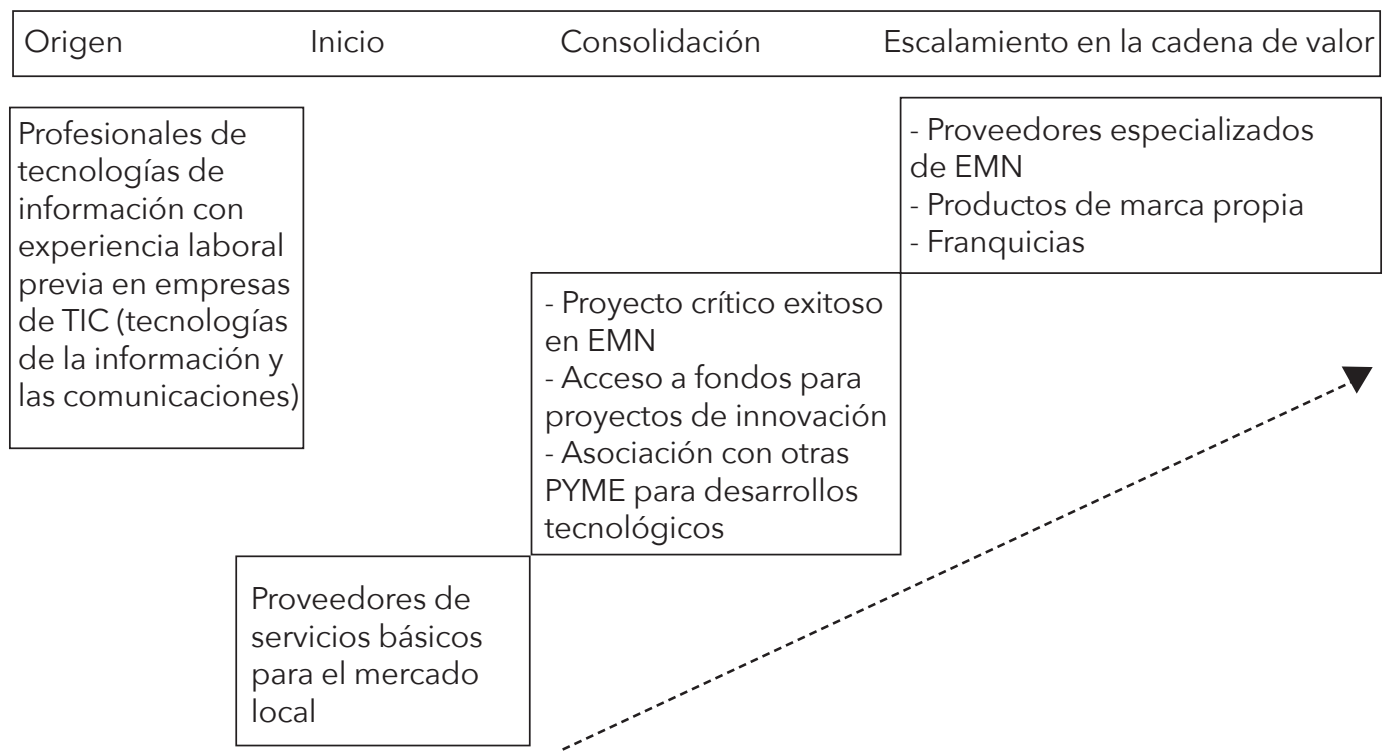

Fuente: elaboración propia. 


\section{Conclusiones}

En este artículo se propone una caracterización de las PYME tecnológicas mexicanas, bajo la hipótesis de que los principales mecanismos de formación y escalamiento de este tipo de empresas se relacionan con las derramas de conocimiento por parte de las EMN, y con la maduración de algunos sistemas regionales de innovación (SRI) en México.

El contexto relevante para la creación de PYME tecnológicas mexicanas se relaciona con la afluencia masiva de IED a México, que durante los últimos 25 años ha contribuido a conformar en México una economía dislocada, en la que se han consolidado algunas actividades muy dinámicas y modernas ligadas a los mercados externos, mientras que otras, orientadas al mercado interno, se mantienen estancadas o en retroceso. En este escenario de polarización, las PYME, que constituyen la gran mayoría de las unidades económicas del país, han quedado por lo general al margen de la modernización y sin vínculos con la economía global. Su propio rezago tecnológico y empresarial, sumado a la ausencia de una política efectiva diseñada para su fortalecimiento, ha provocado que la mayoría de ellas se mantengan ajenas a las cadenas globales de valor.

Sin embargo, un pequeño pero creciente número de PYME locales ha incursionado en actividades intensivas en conocimiento y se han convertido en proveedoras de EMN. En parte, se trata de un efecto de las derramas tecnológicas y de conocimiento de las empresas globales que se han establecido en diversas regiones del país durante el periodo del TLCAN; ya sea a través de desprendimientos tipo spin-off o mediante procesos de aprendizaje interactivo, la relación de estas PYME con las EMN les ha permitido acumular capacidades tecnológicas y empresariales para incursionar como proveedoras de productos 0 servicios de alto valor agregado. En otros casos, la acumulación de capacidades se debe sobre todo a la existencia de algunos entornos regionales donde las redes institucionales y empresariales han conformado sistemas regionales de innovación relativamente maduros y propicios para la formación de empresas locales intensivas en conocimiento.

Entre los resultados relevantes, el estudio destaca el hecho de que en las empresas creadas mediante desprendimientos tipo spin-off, el aprendizaje por interacción con las EMN es el mecanismo más importante para la acumulación de capacidades; por su parte, en las que surgieron como emprendimientos fuera del circuito de las EMN, las relaciones con el entorno institucional (centros de educación superior y de investigación, programas gubernamentales y organismos empresariales) constituyen la principal vía de adquisición de las capacidades tecnológicas y empresariales que les permiten competir en segmentos de alto valor agregado. En ambos casos, estamos ante un fenómeno relativamente reciente, asociado al flujo masivo de IED hacia México durante la vigencia del TLCAN, a la expansión de las EMN en el país y, en general, al incremento de la integración de la economía mexicana a la de Estados Unidos.

A finales de 2019 aún es incierto el futuro del TLCAN y su eventual continuación en el Tratado entre México, Estados Unidos y Canadá (T-MEC). El resultado final dependerá principalmente de su aprobación por el Congreso de los Estados 
Unidos, y su desenlace tendrá impactos específicos en las PYME tecnológicas mexicanas. Al respecto, se pueden conjeturar al menos dos escenarios posibles. En el escenario de que el T-MEC no sea ratificado y se interrumpa la continuidad del TLCAN, las PYME mexicanas proveedoras de partes, equipos, servicios de ingeniería, manufactura avanzada y componentes de alto valor agregado, así como aquellas que han desarrollado innovaciones y marcas propias, podrían verse forzadas a reducir sus actividades. Sin embargo, dado que se trata de empresas que previamente han fortalecido sus capacidades tecnológicas y empresariales, muchas de ellas estarían en condiciones de mantener sus operaciones y de buscar nuevos mercados, tanto en México como en otros países.

Por otra parte, en el escenario de la aprobación del T-MEC y de una continuidad en la política de apertura comercial en la relación de México con Estados Unidos y Canadá, algunas PYME locales que proveen a EMN de partes, componentes y maquinados de bajo valor agregado, estarían en condiciones de identificar oportunidades para fortalecer sus capacidades tecnológicas, innovar, desarrollar productos y servicios de mayor contenido tecnológico, mientras que aquellas PYME locales que proveen servicios intensivos en conocimiento, así como partes y componentes de alto valor agregado podrían crecer, ampliar su mercado y escalar hacia nuevas funciones productivas o incursionar en segmentos de mayor valor agregado en diversas cadenas de valor.

El capítulo 25 del T-MEC establece algunos mecanismos para incrementar la participación de las PYME de los tres países en el comercio regional y promover su competitividad en los mercados globales, así como incentivos para que fortalezcan sus capacidades tecnológicas y de innovación, objetivos que se alinean con otros temas sustantivos del T-MEC, en particular con las reglas de origen y el contenido nacional. Aunque tales mecanismos e incentivos tienen alcances limitados, su inclusión es importante porque supone el reconocimiento de una debilidad de origen en el TLCAN.

En ambos escenarios, ya sea el de ratificación del T-MEC o de terminación del TLCAN sin nuevo acuerdo comercial, es crucial que el gobierno mexicano transite hacia esquemas novedosos y focalizados de apoyo al fortalecimiento de las capacidades tecnológicas, empresariales y comerciales de las PYME locales, que permitan matizar y revertir la polarización económica generada en las últimas décadas.

\section{Referencias}

Alarcón Osuna, M. A., y Díaz Pérez, C. C. (2016). La empresa de base tecnológica y su contribución a la economía mexicana en el periodo 2004-2009. Contaduría y administración, 61(1), 106-126. doi:10.1016/j.cya.2015.09.004

Bair, J., y Gereffi, G. (2001). Local clusters in global chains: the causes and consequences of export dynamism in Torreon's blue jeans industry. World Development, 29(11), 1885-1903. doi: 10.1016/s0305-750x(01)00075-4

Bianchi, C., Glavas, C., y Mathews S. (2017). SME international performance in Latin America: The role of entrepreneurial and technological capabilities. 
Journal of Small Business and Enterprise Development, 24(1), 176-195. doi: 10.1108/JSBED-09-2016-0142

Carrillo, J., y Lara, A. (2005). Mexican maquiladoras: new capabilities of coordination and the emergence of a new generation of companies. Innovation and Economic Development, 7(2-3), 256-273. doi: 10.5172/impp.2005.7.23.256

Carrillo, J., y Zárate, R. (2004). Proveedores en la industria electrónica en Baja California. En J. Carrillo y R. Padilla (coords.), La industria maquiladora mexicana (pp.195-220). México: El Colegio de la Frontera Norte- Universidad de Guadalajara.

Casalet Ravenna, M., Buenrostro Mercado, H. E., y Becerril Posadas, G. (2009). La construcción de las redes de innovación en los clústeres de software en dos regiones mexicanas: Aguascalientes y Nuevo León. En A. Martínez, P. López, A. García y S. Estrada (coords.), Innovación y competitividad en la sociedad del conocimiento (pp. 187-209). México: Plaza y Valdés.

Casalet Ravenna, M., y González, L. (2004). Las tecnologías de la información en las pequeñas y medianas empresas mexicanas. Scripta Nova: Revista Electrónica de Geografía y Ciencias Sociales, 8(21). Recuperado de http:// www.ub.edu/geocrit/sn/sn-170-21.htm

Contreras, O. (2016). El eslabón perdido: pequeñas empresas tecnológicas en las cadenas de valor del TLCAN. En M. Tawil, I. Aguilar, N. Fuentes, J. Le Clerco, S. Núñez y L. Ruano (coords.), Integración en América Latina del Norte (1994-2016) (pp. 401-414). México: El Colegio de México.

Contreras, O. (2000) Empresas Globales, Actores Locales: Producción Flexible y Aprendizaje Industrial en las Maquiladoras. México: El Colegio de México.

Contreras, O., y Carrillo, J. (2012). Las empresas multinacionales como vehículos para el aprendizaje y la innovación de las empresas locales. En A. Bracamonte y 0 . Contreras (coords.), Ciencia, tecnología e innovación para el desarrollo económico (pp. 325-350). Hermosillo: El Colegio de Sonora y Consejo Estatal de Ciencia y Tecnología.

Contreras, O. F., Carrillo, J., y Alonso, J. (2012). Local entrepreneurship within global value chains: a case study in the Mexican automotive industry. World Development, 40(5), 1013-1023. doi: 10.1016/j.worlddev.2011.11.012

Contreras, O., Carrillo, J., y García, M. (2017). Empresas locales en cadenas globales de valor: un estudio de caso en la industria automotriz mexicana. En D. Panigo, A. Gárriz, P. Lavarello (coords.), La encrucijada del autopartismo en América Latina (pp. 374-394). Argentina: CONICET.

Contreras, O., y García, M. (2017). KIBS and NTBF in México: combining GVC and RIS to study market entry mechanisms and upgrading. Revista Debates sobre Innovación, 1(1), 1-14. Recuperado de http://www.uam.mx/altec2017/pdfs/ALTEC_2017_paper_216.pdf

Contreras, O., y Hualde, A. (2012). Empresas multinacionales, aprendizaje tecnológico y desarrollo. Una revisión del debate. En J. Carrillo (coord.), La importancia de las multinacionales en la sociedad global. Viejos y nuevos retos para México (pp. 415-419). México: El Colegio de la Frontera Norte y Juan Pablos Editor. 
Contreras, O., e Isiordia, P. (2010). Local institutions, local networks and the upgrading challenge. Mobilising regional assets to supply the global auto industry in Northern Mexico. International Journal of Automotive Technology and Management, 10(2-3), 161-179. doi: 10.1504/IJATM.2010.032622

Cooke, P. (1992). Regional innovation systems, clusters, and the knowledge economy. Industrial and Corporate Change, 10(4), 945-975.

Cooke, P., Uranga, M. G., y Etxebarria, G. (1998). Regional systems of innovation: an evolutionary perspective. Environment and planning A, 30(9), 15631584. doi: 10.1068/a301563c

Dini, M., y Stumpo, G. (2004). Pequeñas y medianas empresas y eficiencia colectiva: estudios de caso en América Latina. México: CEPAL y Siglo XXI Editores.

Dini, M., y Stumpo, G. (2011). Políticas para la innovación en las pequeñas y medianas empresas en América Latina. Santiago de Chile: CEPAL.

Dussel Peters, E. (2012). Inversión extranjera directa, especialización territorial e innovación en México (1994-2007). En J. Carrillo (coord.), La importancia de las multinacionales en la sociedad global (pp. 105-132). México: El Colegio de la Frontera Norte y Juan Pablos Editor.

Frederick, S., y Gereffi, G. (2011). Upgrading and restructuring in the global apparel value chain: why China and Asia are outperforming Mexico and Central America. International Journal of Technological Learning, Innovation and Development, 4(1-3), 67-95. doi: 10.1504/IJTLID.2011.041900

Freeman, C. (1987). Technology Policy and Economic Performance: Lessons from Japan. Londres: Pinter Publishers.

Fuentes, C. de, y Dutrénit, G. (2008). Diferencias de los mecanismos de derramas de conocimiento en dos localidades mexicanas. Economía y Sociedad, 14(22), 47-69. Recuperado de http: / /www. redalyc.org/html/510/51002203/ index.html

Gereffi, G. (1994). The organization of buyer-driven global commodity chains: how U. S. retailers shape overseas production networks. En G. Gereffi y M. Korzeniewicz (coords.), Commodity Chains and Global Capitalism (pp. 95122). Estados Unidos: Praeger Publishers.

Gereffi, G., y Fernandez-Stark, K. (2011). Global value chain analysis: a primer. Estados Unidos: Center on Globalization, Governance \& Competitiveness (CGGC). Recuperado de https://www.researchgate.net/profile/Karina_Fernandez-Stark/publication/265892395_Global_Value_Chain_Analysis_A_Primer/links/54218b000cf274a67fea984b.pdf

Gereffi, G., y Fernandez-Stark, K. (2016). Global Value Chain Analysis. A primer. Duke Center on Globalization, Governance and Competitiveness. Recuperado de https://www.researchgate.net/profile/Gary_Gereffi/publication/305719326_Global_Value_Chain_Analysis_A_Primer_2nd_Edition / links/579b6f0708ae80bf6ea3408f/Global-Value-Chain-Analysis-A-Primer2nd-Edition.pdf

Gereffi, G., Humphrey, J., y Sturgeon, T. (2005). The governance of global value chains. Review of international political economy, 12(1), 78-104. Recuperado de http://www.fao.org/fileadmin/user_upload/fisheries/docs/GVC_Governance.pdf 
Gereffi, G., y Kaplinsky, R. (2001). The value of value chains: spreading the gains from globalization. Special issue of the IDS Bulletin, 32(3), 1-7. Recuperado de http://el.doccentre.info/eldoc/u00_/the\%20value\%20of\%20 value\%20chains1.pdf

Gereffi, G., y Korzeniewicz, M. (coords.). (1994). Commodity Chains and Global Capitalism. Westport, Connecticut, Estados Unidos: Praeger Publishers.

Giuliani, E., Pietrobelli, C., y Rabellotti, R. (2005). Upgrading in global value chains: lessons from Latin American clusters. World Development, 33(4), 549-573. doi: 10.1016/j.worlddev.2005.01.002

Hecker, D. E. (2005). High-technology employment: a NAICS-based update. Monthly Labor Review, 128(7), 57-72. Recuperado de: https://www.bls. gov/opub/mlr/2005/07/art6full.pdf

Hualde, A., y Gomis, R. (2007). PYME de software en la frontera norte de México: Desarrollo empresarial y construcción institucional de un cluster. Problemas del Desarrollo. Revista Latinoamericana de Economía, 38(150), 193212. doi: 10.22201/iiec.20078951e.2007.150.7676

Humphrey, J., y Schmitz, H. (2004). Chain governance and upgrading: taking stock. En Hubert Schmitz (ed.), Local Enterprises in The Global Economy Issues of Governance and Upgrading. Cheltenham: Edward Elgar.

Instituto Nacional de Estadística y Geografía (INEGI). (2014). Sistema de Clasificación Industrial de América del Norte, México SCIAN 2013. México: Instituto Nacional de Estadística y Geografía. No. 588. Recuperado de http://www. inegi.org.mx/est/contenidos/proyectos/scian/presentacion.aspx

Instituto Nacional de Estadística y Geografía (INEGI). (2018). Directorio Estadístico Nacional de Unidades Económicas: DENUE Interactivo 03/2018: Documento Metodológico. México: Instituto Nacional de Estadística y Geografía. Recuperado de http://internet.contenidos.inegi.org.mx/contenidos/ Productos/prod_serv/contenidos/espanol/bvinegi/productos/nueva_estruc/702825100841.pdf

Jurowetzki, R., Lema, R., y Lundvall, B.-A. (2018). Combining innovation systems and global value chains for development: towards a research agenda. The European Journal of Develpoment Research, 30(3), 364-388. doi: 10.1057/s41287-018-0137-4

Kile, C. O., y Phillips, M. E. (2009). Using industry classification codes to sample high-technology firms: Analysis and recommendations. Journal of Accounting, Auditing \& Finance, 24(1), 35-58. doi: 10.1177/0148558X0902400104

Klepper, S., y Thompson, P. (2005). Spinoff entry in high-tech industries: motives and consequences. En F. Malerba y S. Brusoni (eds.), Perspectives on Innovation (pp. 187-218). Reino Unido: Cambridge University Press.

López de Alba, P. (2014). El modelo de articulación productiva, base de los sistemas de innovación en el estado de Guanajuato. En M. Zavala (coord.), Investigación para la transformación social. Experiencias de investigación vinculada (pp. 75-91). México: Universidad de La Salle.

Lundvall, B.-A. (1992). National Systems of Innovation: Towards a Theory of Innovation and Interactive Learning. Londres: Pinter Publishers. 
Lundvall, B.-A. (2007). National innovation systems-analytical concept and development tool. Industry and Innovation, 14(1), 95-119. doi: $10.1080 / 13662710601130863$

Malerba, F., y Nelson, R. (2011). Learning and catching up in different sectoral systems: evidence from six industries. Industrial and Corporate Change, 20(6), 1645-1675.

Mendoza, J., y Valenzuela, A. (2014) Aprendizaje, innovación y gestión tecnológica en la pequeña empresa: un estudio de las industrias metalmecánica y de tecnologías de información en Sonora. Contaduría y Administración, 59(4), 253-284. doi: 10.1016/S0186-1042(14)70162-7

Merton, R. K. (1968). Social Theory and Social Structure. Estados Unidos: Macmillan.

Muller, E., y Doloreux, D. (2009). What we should know about knowledge-intensive business services. Technology in Society, 31(1), 64-72. doi: 10.1016/j. techsoc.2008.10.001

Pietrobelli, C., y Rabellotti, R. (2006). Upgrading to Compete Global Value Chains, Clusters, and SMEs in Latin America. Washington: Harvard University Press. Recuperado de https: / /ssrn.com/abstract=1551498

Pietrobelli, C., y Rabellotti, R. (2011). Global value chains meet innovation systems: are there learning opportunities for developing countries? World Development, 39(7), 1261-1269. doi: 10.1016/j.worlddev.2010.05.013

Portes, A. (2004). La sociología en el continente: convergencias pretéritas y una nueva agenda de alcance medio. Revista Mexicana de Sociología, 66(3), 447-483.

Secretaría de Economía, Dirección General de Inversión Extranjera. (2019). Información estadística de flujos de IED hacia México por entidad federativa. https://datos.gob.mx/busca/dataset/informacion-estadistica-de-la-inversion-extranjera-directa

SEDESOL, CONAPO, INEGI. (2012). Delimitación de las zonas metropolitanas de México. México: SEDESOL, CONAPO, INEGI. Recuperado de http://www. conapo.gob.mx/es/CONAPO/Delimitacion_zonas_metropolitanas_2010_Capitulos_I_a_IV

Smelser, N., y Swedberg, R. (eds.). (2005). The Handbook of Economic Sociology. Princeton University Press.

Trippl, M. (2006). Cross-Border Regional Innovation Systems. Viena: Institut für Regional-und Umweltwirtschaft, WU Vienna University of Economics and Business (SRE Discussion Papers, 2006/05).

Valenzuela Básaca, N. A., y Bracamonte Sierra, A. (2014). Microsistemas de innovación: parques tecnológicos en Sonora. En A. Bracamonte y O. Contreras, (coords.), Tecnología y competitividad. Conceptos y experiencias prácticas (pp. 137-176). Hermosillo: El Colegio de Sonora.

Vera-Cruz, A. O., y Dutrénit, G. (2005). Spillovers from MNCs through worker mobility and technological and managerial capabilities of SMEs in Mexico. Innovation, 7(2-3), 274-297. doi: 10.5172/impp.2005.7.2-3.274

Villavicencio, D., y Arvanitis, R. (2001). Las capacidades de innovación en la industria química en México. En G. Dutrenit et al. (coords.), Sistema Na- 
cional de Innovación Tecnológica: temas para el debate en México (pp. 379394). México: UAM.

Yuhua, Z. (2014). Integrating SMEs into global value chains: policy principles and best practices. Issues Paper No. 6. APEC Policy Support Unit, APEC, May. Zevallos, E. V. (2003). Micro, pequeñas y medianas empresas en América Latina. Revista de la CEPAL, 79, 53-70. 